\title{
Heuristic threshold for Histogram-based Binarization of Grayscale Images
}

\section{RESUMEN}

The development of a Virtual Lab to perform experiments on histogram-based binarization of grey-leveled images is reported. With the aim of automatizing the binarization process, a Heuristic Binarization Threshold is introduced. Once the histogram of a grayscale input image is obtained, the module calculates a Heuristic Threshold by taking the weighted average of the foreground grey levels of the image. Next those pixels in input image whose grey levels are above this threshold are highlighted. Although still not experimentally optimum, this heuristic threshold provides a first approximation towards automatic binarization of greyscale images.

Palabras clave: automatic binarization, digital image processing, grayscale histogram, thresholding

UMBRAL HEURÍSTICO PARA BINARIZACION VÍA HISTOGRAMA DE IMÁGENES EN NIVELES DE GRIS

\section{ABSTRACT}

Se reporta el desarrollo de un laboratorio Virtual basado en histograma para llevar a cabo experimentos sobre binarizacion de imágenes en niveles de gris. A fin de automatizar el proceso de binarizacion, se introduce un Umbral Heurístico de Binarizacion. Después de obtener el histograma de una imagen en niveles de gris el modulo calcula el umbral heurístico, extrayendo el promedio ponderado de los niveles de gris de primer plano de la imagen. A continuación se resaltan los niveles de gris en la imagen que están por encima del umbral heurístico. Aunque aún no experimentalmente óptimo, el umbral heurístico proporciona una primera aproximación hacia la binarizacion automática de imágenes en niveles de gris.

Keywords: binarizacion automática, histograma de niveles de gris, umbral procesamiento digital de imágenes

\section{INTRODUCTION}

When the information of an image is contained in the shape of the objects within the image rather than in their color, it is convenient to convert the image to strict black and white, this is, to binarize the image. This need arises not only in automatic digital image processing applications but also when a professional (may be a physician) makes a diagnostic based on plain image visualization.

Binarization of images is the indispensable pre-processing in pattern recognition applications of digital image processing [16]. Binarized (strict black \& white) images are used in those applications focusing on the shape of the objects in the image under scrutiny, like quality control, security and defense systems, medical diagnostics, etc.

Binary images are used in quality control in modern industrial plants, for automatic shape defect detection. An example may be the case of chocolate factories where pattern recognition techniques may be used to detect flaws in the shape of the chocolates. If chocolates must keep a rectangular or squared shape, a technique that may be implemented to achieve this shape control may be based on the Polar Hough Transform $[2,7,8,9,10,13,15]$, which performs on strictly black and white (binary) images. Other Hough-transform based algorithms may also be used to detect the perfection of circular and elliptical shapes. Another example may be the shape control in plastic material pieces coming out of an injector machine making them.

Pattern recognition applications in defense systems operate upon binary images. These applications might make use of algorithms based on Invariant Moments [2,11,12,14,15,16], which require a pre-processing to binarize grayscale images before extracting information from them.

\section{HISTOGRAMS}

The histogram [1-4] of a grayscale digital image gives the number of pixels per gray level in the image. In this way the histogram gives also information about the probability of finding a given gray-level in the image, the higher the number of pixels for a given gray level, the higher the probability of finding that gray level in the image, and vice versa.

Director, VirtualDynamicsSoft: Science \& Engineering Virtual Labs. Faculty of Physics at UNMSM. Director@VirtualDynamics.Org www.VirtualDynamicsSoft.com 


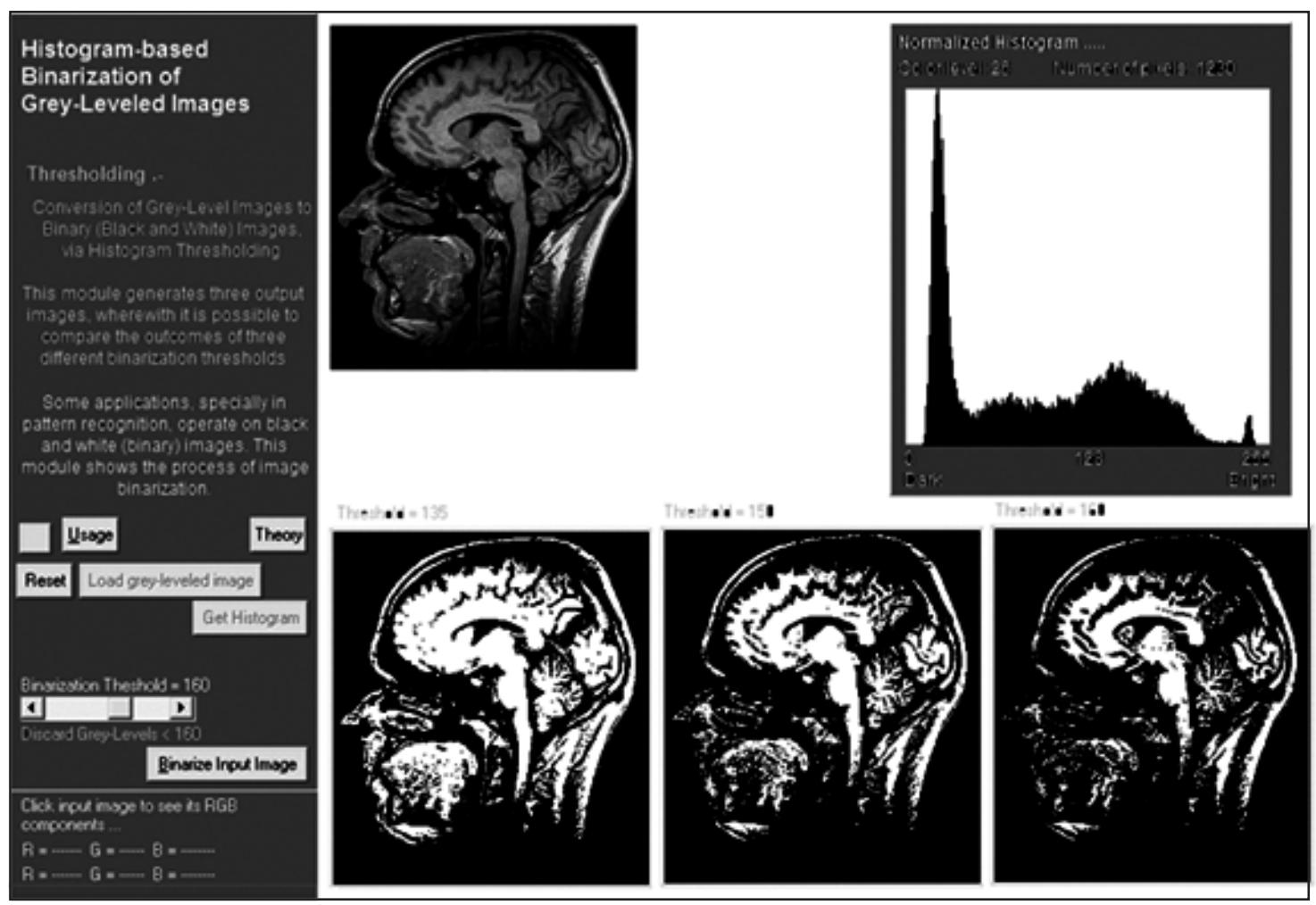

Figure 1 (Top): The input image, a NMR grey-levelled image of a human head. (Right): The corresponding histogram, where the user sets the binarization threshold. (Bottom): Three binarizations for thresholds of 135, 150 and 160, respectivelly.

In order to generate the grey-level histogram [1516 ] of an image, a census to make a list of number of pixels per grey level must be made (see Fig 2). The histogram is the plotting of number of pixels versus grey level. In this way the horizontal axis of the histogram ranges from 0 (dark levels) through 255 (clear color levels), and the vertical is the pixel population in each grey level. An option to execute this census is, for example, sweeping top-down and left-right the image and counting the number of pixels having every one of the $\mathbf{2 5 6}$ gray levels from 0 through 255 .

\section{HISTOGRAM EQUALIZATION}

A histogram has been equalized $[15,16]$ when it has been normalized between 0 and 1 , with 0 representing black and 1 signifying white. In this way the gray levels may be regarded as random quantities in the interval from 0 to 1 .

In the Virtual Lab being reported here, the histogram is rendered on screen and as the mouse hovers over it, the grey-level under the mouse and its population is reported. In order to fix the binarization threshold, one of these grey-levels on the histogram must be mouse clicked, or selected by displacing a scroll bar.
Figura 2.

\begin{tabular}{|c|c|c|}
\hline $\begin{array}{l}\text { Number of } \\
\text { Gray Level } \\
0 \\
1 \\
2 \\
3 \\
4 \\
5 \\
6 \\
7 \\
: \\
\vdots \\
250 \\
251 \\
252 \\
253 \\
254 \\
255\end{array}$ & $\begin{array}{l}\text { xeis per gray level. } \\
\text { Pixels } \\
91458 \\
2995 \\
1264 \\
996 \\
723 \\
566 \\
443 \\
341 \\
: \\
: \\
558 \\
827 \\
1199 \\
1871 \\
3129 \\
37786\end{array}$ & $\begin{array}{l}\text { An example of a table of } \\
\text { pixel population per grey } \\
\text { level: } \\
\text { The grey levels run from } 0 \\
\text { through } 255 \text { and, every } \\
\text { grey level is associated to } \\
\text { the number of pocels in } \\
\text { input image having that } \\
\text { level. } \\
\text { The histogram is made by } \\
\text { plotting population versus } \\
\text { grey level. } \\
\text { In a normalized histogram } \\
\text { the grey levels run from } 0 \\
\text { through } 1 \text {. }\end{array}$ \\
\hline
\end{tabular}

\section{THRESHOLDED BINARIZATION}

In order to achieve the grayscale image binarization, the image may be scanned top-down and left-right, and those pixels in the image whose grey-levels are above the predefined threshold must be highlighted [14], by for example, showing them in white colour on a black background, or vice versa. 


\section{HEURISTIC THRESHOLDING}

Setting a binarization threshold is not an easy task because it depends on the image and on the requirements of the work being carried out. A computer vision system may require very well defined edges of solid objects, while a physician might need a visualization of the soft tissues in the human body.

Once the histogram of an input image has been generated, it is not so easy to decide which grey level to use as a binarization threshold. Usually grayscale images have dark colours (especially black) as the background and clear colours (especially white) as foreground. Experimentally, when the weighted average grey level was used as a binarization threshold the resulting binary image was not as good as expected. The same happened when taking as a threshold the most populated grey level.

A heuristic binarization threshold may be obtained by automatically discarding the background dark grey levels; let's say from 0 through 40, and taking the weighted average of the remaining levels as the binarization threshold. In this way, the binarization module introduces as a suggested threshold, the
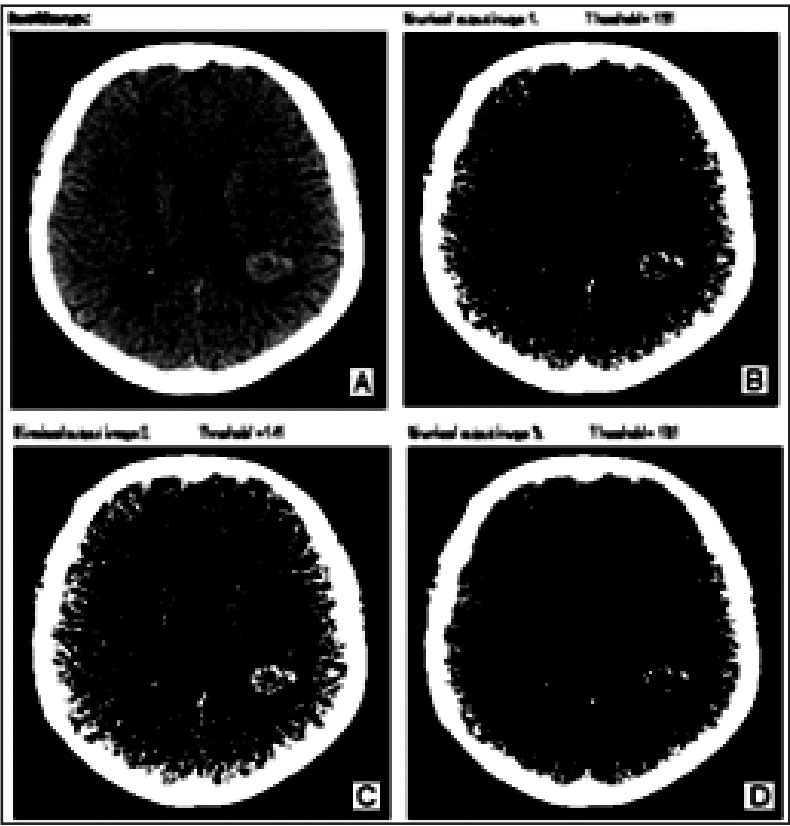

Figure 3 (A) Input image showing a brain tumor in the parietal lobe. (B) Binarized image obtained with the heuristic threshold $(T=151)$, Images $(C)$ and $(D)$ are binarizations corresponding to thresholds of $T=141$ and has been obtained with the heuristic threshold. Binarized images show only the poxels above binarization threshold, pixels below it are discarded. weighted average of the assumed foreground grey levels, those from 41 through 255. Many images were tested with this heuristically obtained threshold and the results were not always those expected, however the obtained threshold may be considered a first approximation towards the best possible binarization threshold.

\section{RESULTS}

Figure 1 Displays a panoramic view of the developed module. The input image at the top is a side view of a Nuclear Magnetic Resonance (NMR) greyscale image of a human head. On the right side appears the corresponding normalized histogram, where the user clicks with the mouse the selected level (Threshold) for binarization. At the bottom, three binarizations are shown, being the first at the left, the automatically obtained binarization with a heuristic threshold of 135; the other two binarizations shown correspond to manually fixed 150 and 160, thresholds respectively.
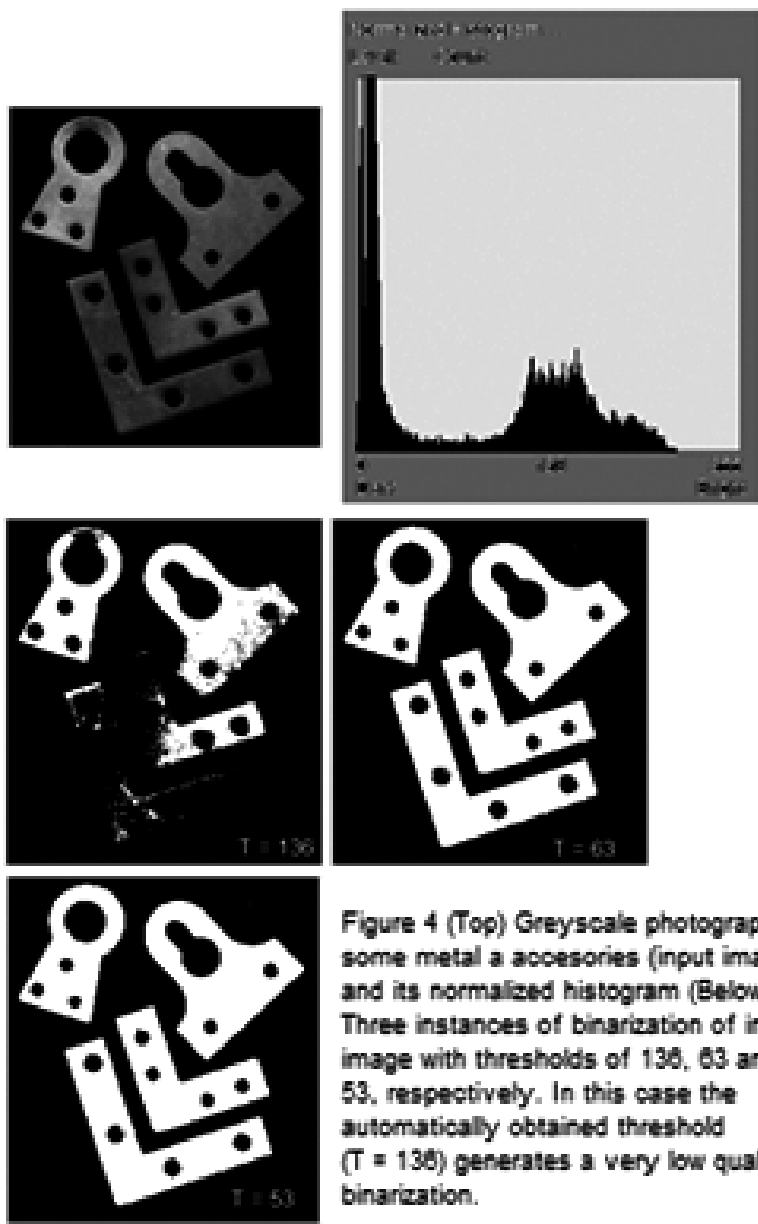

Figure 4 (Top) Greyscale photograp of some metal a accessories (input image) and its normalized histogram (Below); Three instances of binarization of input image with thresholds of 136, 63 and

53. respectively. In this case the automatically obtained threshold $(T=136)$ generates a very low quality binarization. 
In Figure 3 the binarization of the image of a tumor in the brain is shown. In this case the binary image best displaying the profile of the tumor was obtained with the automatically calculated heuristic threshold. With the aim on allowing comparison, Figure 3 displays also binarizations associated with thresholds of 10 above and below the heuristic threshold. It can be seen that result $C$ highlights too many pixels in the image, generating confusion with tissue pixels around the tumor, while result $D$ focuses very few pixels.

Figure 4 displays the binarization of an image displaying some metal accessories. In this case, the input image at the top left is a grey-level photograph of some metal accessories. The corresponding normalized histogram is displayed at the top right. Then three instances of the binarization of the input image are shown below the input image. The corresponding thresholds are 136, 63 and 53, respectively. In this case the automatically obtained heuristic threshold is 136 , the other two thresholds were selected by mouse clicking the histogram. As it can be seen in Figure 2, different thresholds generate binary images of different qualities, and obviously in this case the best binary image has been manually obtained with a threshold of 53 . In this case the automatically obtained heuristic threshold generates a poor quality binarization.

\section{CONCLUSIONS}

A virtual lab (a software module) to achieve a modulated binarization of a grayscale image, based on histogram thresholding, has been devised. The user of the module sets the binarization threshold by mouse clicking the desired gray level in the input image histogram, which is rendered on screen. With a virtual lab like the one being reported, the user may test several binarization thresholds very easily and in a very short time.

With the aim on automatic thresholding, a heuristic binarization threshold based on the weighted average of the foreground grey levels in the image has been introduced. This not always generated the ideal binarization of the input image, however it may be regarded as a first approximation towards a suitable binarization threshold.

\section{REFERENCES}

[1] Gonzales R, Woods R. (2008). Digital Image Processing (Pearson-Prentice Hall), USA.

[2] Gonzales R, Woods R. (1992). Digital Image Processing (Addison - Wesley), USA.
[3] Schalkoff R. J. (1989). Digital Image Processing and Computer Vision, Willey, USA.

[4] Jahne B. (1995). Digital Image Processing, Springer-Verlag, USA.

[5] Chen C.H., Pau L. F. Wang P.S.P. (1995). Handbook of Pattern Recognition \& Computer Vision, World Scientific, USA

[6] Jhane B. (2004). Practical handbook on image processing for scientific and technical applications, CRC Press, USA.

[7] Montenegro Joo, J. (1994). GeometricTransformations Invariant Pattern Recognition in the Hough Space (Doctoral thesis project, USP, Sao Carlos, Brasil).

[8] Montenegro Joo, J. (1998). A Polar-HoughTransform-based Algorithm for the Translation, Orientation and Size Scale Invariant Pattern Recognition of Polygonal Objects. University Microfilms International, UMI Microform LD 0376.

[9] Montenegro Joo, J. (2002). Invariant Recognition of Rectangular Biscuits through an Algorithm Operating exclusively in Hough Space. Flawed Pieces Detection. Revista de Investigación de Física, Vol. 5, UNMSM.

[10] Montenegro Joo,J. (2007). Hough-Transform based Automatic Invariant Recognition of Metallic Corner-Fasteners. JMJ, Industrial Data, Vol. 10 - N. ${ }^{\circ} 1$.

[11] Montenegro Joo,J. (2003). Improved-InvariantEdge Moments without Object-Edge Tracing. Rev. de Electrónica, 12, UNMSM.

[12] Montenegro Joo, J. (2005). Knowing-How on Boundary Geometric Moments. Revista de Electronica, 16, UNMSM.

[13] Montenegro Joo, J. (2006). Hough-transform based algorithm for the automatic invariant recognition of rectangular chocolates. Detection of defective pieces. Industrial Data, Vol 9, N. ${ }^{\circ} 2$.

[14] Montenegro Joo, J. (2006). Boundary Geometric Moments and its application to automatic quality control in the industry. Industrial Data, Vol. 9, N. ${ }^{\circ} 1$.

[15] Montenegro Joo J. (2006). Imagery: Virtual Lab for Computer-Aided training on Digital Image Processing. RISI, Vol. 3, N. ${ }^{\circ} 4$.

[16] Montenegro Joo J. (2009). Imagery 37: Digital Image Processing Virtual Lab., V International Conference on Multimedia and ICT in Education (m-ICTE 2009), Lisboa, Portugal. 